\title{
CAUSALITY IN MODELS OF THERMAL PROCESSES IN SHIP ENGINE ROOMS WITH THE USE OF BOND GRAPH (BG) METHOD
}

\author{
Marian Cichy \\ Zbigniew Kneba \\ Jacek Kropiwnicki \\ Gdansk University of Technology, Poland
}

\begin{abstract}
With a single approach to modeling elements of different physical nature, the method of Bond Graph $(B G)$ is particularly well suited for modeling energy systems consisting of mechanical, thermal, electrical and hydraulic elements that operate in the power system engine room. The paper refers to the earlier presented [2] new concept of thermal process modeling using the $B G$ method. The authors own suggestions for determining causality in models of thermal processes created by the said concept were given. The analysis of causality makes it possible to demonstrate the model conflicts that prevent the placement of state equations which allows for the direct conduct of simulation experiments. Attention has been drawn to the link between the energy systems models of thermal processes with models of elements of different physical nature. Two examples of determining causality in models of complex energy systems of thermal elements have been presented. The firs relates to the electrical system associated with the process of heat exchange. The second is a model of the mechanical system associated with the thermodynamic process.
\end{abstract}

Keywords: Bond Graph (BG) method, causality, ship engine room, energy system modeling

\section{INTRODUCTION}

The social growth of expectations in terms of increasing the efficiency of machinery and equipment as well as reducing greenhouse gas emissions and air pollutants forced in recent years the intensification of research and development to improve the efficiency of heat exchange processes $[4,18]$, the structural improvement of working machines and power equipment $[7,14,16,24]$, improving the quality of diagnostics of these devices $[11,12]$ and the improvement of efficiency of the manufacturing process of machine elements $[6,9]$. One of the more complex energy systems, which is covered by the above-mentioned development work is a ship's engine room.

A ship engine room is an energy system which includes machinery and equipment whose task is to convert chemical Energy into mechanical Energy, electrical and thermal quantities and specifications to ensure the correct operation of receivers necessary for the fulfillment of the assumed unit functions, in all states of operation and the variable external conditions. Marine vessels are characterized by the autonomy of energy, which means that all the energy needed must be met by using fuel consuming elements like main engines, auxiliary boilers, etc. . The proper design, as well as the operation of such a system of energy requires a comprehensive examination of thermal, mechanical and electric processes.

The method of Bond Graphs (BG) which is the core of this study was initiated by Paynter [20] and has been developed in a number of monographs $[1,3,10,26,27,28]$ and publications $[5,8,13,15,17,19,21,22,23,25]$. Thanks to a singular approach to modeling elements of different physical nature, this method is especially suited for modeling energy systems consisting of mechanical, thermal, electrical and hydraulic elements which operate in the power system of an engine room.

The flow of Energy between the individual components of the Energy system is marked with graphs (half-arrow) automatically introducing information about the direction of the flow. The energy flow is always described by two parameters of energy: metastatic potential (e) and generalized flow $(f)$. In the case of the internal combustion engine, 
the parameters characterizing the energy receiver to be transmitted are respectively $M_{o}(e)$ and $\omega(f)$. The product of Energy parameters ( $e$ and $f$ ) must always give the dimension of power.

Models of Bond Graphs composed of external elements: (fig. 1): $S$ (source of energy), $L$ (kinetic energy storage), $C$ (potential energy storage), $R$ (dissipative element) and the internal components: energy converters, node $\mathbf{0}$ of the same generalized potential and nodes 1 of the same generalized flow. The energy sources present in the modeled object, according to the formalism of BG may exist in two forms: as a source of potential: $S E$ (systemic independent potential is a function of time), or as a source of flow: $S F$ (systemic movement is an independent function of time).

The model itself in the form of BG reflects clearly the dynamic structure of the object and can be easily modified, but does not allow for the direct conduct simulation experiments [3]. The BG model allows for a „manual” or automatic generation of equations of state, which means creating a mathematical model of cause and effect.

Using the causality discussed in this paper, one can describe the relationship between metastatic potential (e) and generalized flow (f). By convention, the designation of BG causality edge is represented by short dash perpendicular line at one end of the Edge (fig. 4). The position of this line indicates which value $e$ or $f$ is a dependent and which independent value (e.g. fig. 4 a). Such value is $\mathrm{T}(\mathrm{t})$ ). The analysis of causality makes it possible to demonstrate conflicts that prevent the placement of state equations. These conflicts can be of two types:

- a conflict related to the use of improper or inadequate energy source for the energy source used, as of the internal structure of the model,

- a conflict caused by the introduction of interdependent power accumulators into the model.

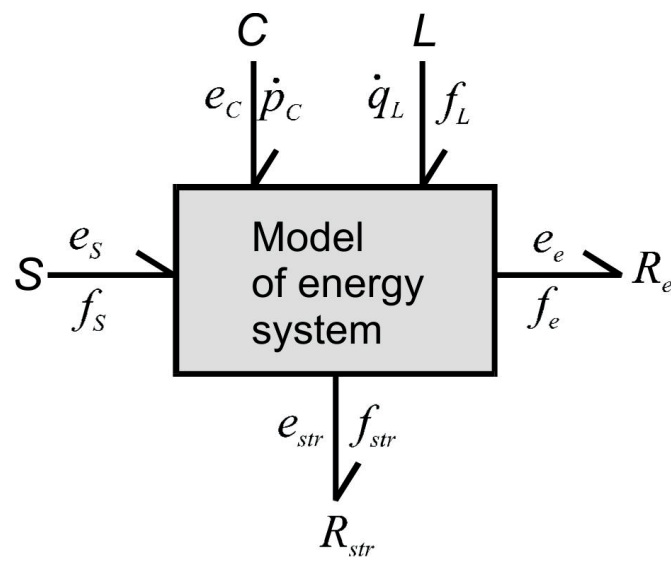

Fig. 1 Diagram and designation of BG model Energy system to determine the efficiency of: $e$ - generalized potential, $f$-generalized flow, $p$-generalized momentum, $q$-generalized displacement [3]

The basic theory of BG concept of causality and use of it to analyze the BG models i clearly shown $[1,3,10,26,27]$ in the construction of models of mechanical, electrical and hydraulic processes. Doubts arise when creating BG models of thermal processes, especially regarding the new elements introduced into these models as a dual battery $[1,27]$ (2-port) or the modeling of thermal processes using so-called „pseudo Bond Graphs" [1, 3, 8, 10, 26, 27]. In [2, 3] a new method of modeling thermal processes has been presented using only elements introduced in the basic BG theory form modeling of mechanical, hydraulic and electrical processes. In these works the analysis of causality was deliberately not used since according to the theory proposed in separate models of thermal processes, there was no possibility of a conflict of causality [3].

In [2] it was found that the Energy storing models of thermal processes, as in the basic theory, should be in the form of a graph (bond) with a single node (1-port). The concept of an energy transducer has been introduced [2, 3] (fig. 2). In the basic theory of BG the transducer exists in the form of a transformer or gyrator. In general theory of thermodynamics and heat exchange there are physical concepts that could justify the introduction of such a distribution of energy converters. Introduced in [2] energy converters must comply only with the basic theory of the BG relationship (1) of the net amount of energy flow in any multiple node (multiports), in which the $n$ graphs (bonds).

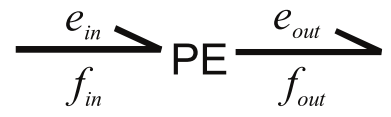

Fig. 2. Power converter in BG model [2]

$$
\sum_{i=1}^{n} e_{i} f_{i}=0
$$

When modeling the extracted thermal processes, the available literature does not provide a specified example where a conflict of causality would be shown. Numerous examples of creating equations of state based on the BG model in $[2,3]$ showed that the determination of causality can only serve to illustrate the flow of energy. However, the need for analysis of causality exists only when the systems are modeled, in which the nest thermal processes taking place relate to processes of different physical nature such as the mechanical or electrical one. In real power systems such links always take place. Simulation studies using BG model can be carried out when the graphic model allows to generate equations of state in the form of:

$$
\dot{\mathbf{X}}=\mathbf{f}(\mathbf{X}, \mathbf{U})
$$

where: $\mathbf{X}$ - vector of state variables,

$\mathbf{U}$ - vector of controls.

The conflict of causality prevents the arrangement of the equation in the form of (2). 


\section{CAUSALITY IN BG ELEMENTS IN THERMAL PROCESSES MODEL}

The basic elements of BG, which should clearly define the causality are the heat energy accumulators and energy sources. As shown in [2] the thermal processes, there is only one type of energy storage accumulator wherein the state variable characterizing is the temperature of the kinetic energy of the molecules. There is no physical evidence to assign two kinds of causality to heat accumulators just as it has been, for example, in the mechanical processes, namely integral and differential causality. According to this reason, causality of heat accumulator is marked clearly as shown in Fig. 3. According to the general theory of BG it is causality of the generalized potential of the kinetic energy accumulator.

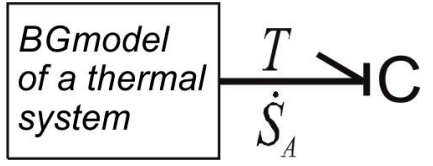

Fig. 3. Determination of causality of thermal energy accumulator in the $B G$ model

The general form of the dependence defining the flow of the accumulated heat is presented by the relation:

$$
T \dot{S}_{A}=\dot{E}_{A}=\frac{\mathrm{d}\left(C_{v} m T\right)}{\mathrm{d} t}
$$

For many cases, for example, heat conduction, it can be assumed that the product is $C_{v} m=$ const, then $\dot{E}_{A}=C_{v} m \dot{T}$.

In work [2] various types of heat sources have been shown, which are graphically shown in Fig. 4 . In the general theory of BG $[1,3,10,27]$ the causality of energy sources is uniquely determined for a source of potential or sources of movement. Case „b” on Fig. 4 shows an energy source in the form of mass flow of agent $\dot{m}$ of enthalpy $i$. There is here the special case where both generalized parameters can be independent time functions. Fig. 4 indicates, as example, a generalized flow causality. Determination of the energy gates, which are regarded arbitrarily as a source of Energy, depends on the purpose served by the built model. For example, in the electric power network as an energy source may be indicated the energy flux in the fuel supplied to the boiler steam turbine, or otherwise as an energy source one can treat the electrical energy received from the generator current. In the first case, causality will be designated as the source of the flow, and in the second one, as source of potential.

$$
\begin{aligned}
& \left.\mathrm{SE}_{\mathrm{T}} \frac{T}{\dot{S}} \longrightarrow T=T(t) \quad \mathrm{a}\right)
\end{aligned}
$$

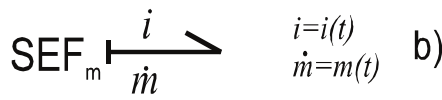

$$
\begin{aligned}
& \left.\mathrm{SF}_{Q} \frac{e_{E}}{f_{E}} \quad e_{E} f_{E}=Q(t) \quad \mathrm{c}\right) \\
& \left.\mathrm{SF}_{\mathrm{V}} \frac{p}{\dot{V}}>\quad \dot{V}=V(t) \quad \mathrm{d}\right)
\end{aligned}
$$

Fig. 4. Sources of Energy and determination of causality in the thermal processes $B G$ model

\section{CAUSALITY IN COMPLEX BG MODELS}

As a first example of a complex system in which the thermal processes are associated with processes of different physical nature an electric circuit with a resistor will be considered (fig. 5) in which the heating and heat exchange with the environment takes place. The BG model of these processes is shown in Fig. 6 and consists of the electrical circuit model and the model of the heating system. The source of power is the voltage generator $V(t)$ of the internal resistance $R_{s}$. The external resistor $R$ is the thermal energy accumulator of mass $m$, specific heat $C_{v}$ and surface of heat exchange with environment $F$.

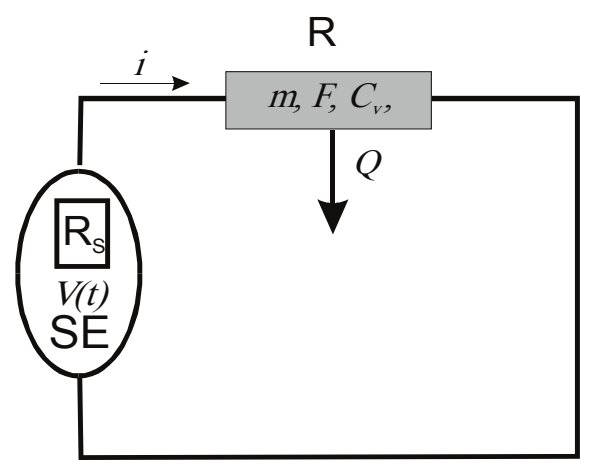

Fig. 5. The electrical circuit in conjunction with a thermal process

The output equation of the state follow directly from the BG model shown in Fig. 6:

$$
\dot{E}_{A}=\left(\frac{V(t)}{R_{S}+R}\right)^{2} R-\dot{S}_{R} T
$$

In this equation, the flow of heat exchange with the environment is determined by the relationship: 


$$
\dot{S}_{R} T=F \alpha\left(T-T_{O}\right)
$$

where: $\alpha$ - heat transfer coefficient of the resistor surface area $F$ and temperature $T$ to ambient temperature $T_{O}$.

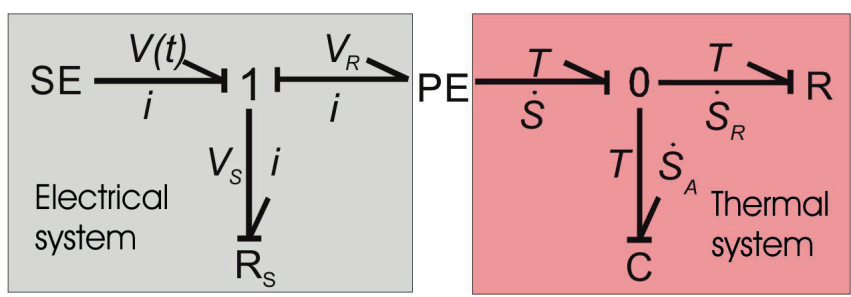

Fig. 6. BG model of related systems: electrical and thermal

As already mentioned, the determination of causality of the BG model of the electrical system in Fig. 6 has been made according to the general principles of the BG theory $[3,10,27]$. According to these rules, the causality scattering element $R$ can be arbitrary. Assuming the causality of heat accumulator $\mathrm{C}$ according to the above accepted rule, the causality of other thermal model graphs can be marked in different ways as shown in Fig. 6 and 7. In any of these cases, there is no conflict of causality. Assuming the rules introduced above, the causality of the PE energy transducer results from the adopted arbitrary causality of dissipation elements and can be gyrator causality (fig. 6) or of the transformer (fig. 7).

a)

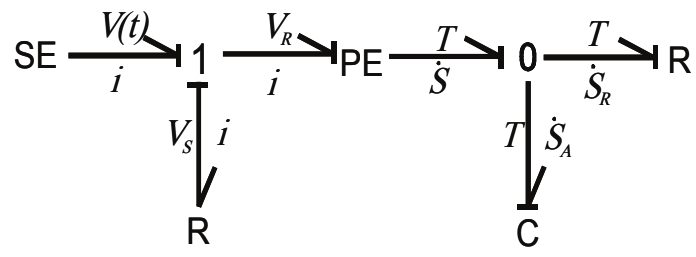

b)

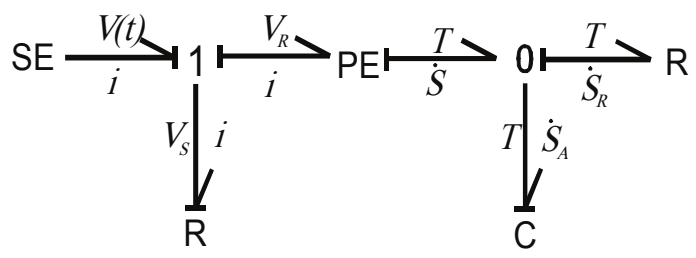

Fig. 7. Two additional versions of the possible designation of BG causality model from Fig. 6

The second example concerns the relationship of the mechanical system in which the change in volume $V$ by changing the predetermined piston speed $v(t)$, of the thermodynamic system, wherein the solid mass of gas $m$ is in variable volume $V(t)$ (fig. 8). The existence of friction force $F_{t}$ was assumed in a piston cylinder and the introduction of the heat flux $Q(t)$ to the gas medium. The BG model of the related system indicating causality is shown in Fig. 9. In this system, there are two energy converters. The first one is a transformer (TR) with unambiguous causality, the second a transduce in a thermal system $\left(\mathrm{PE}_{\mathrm{vs}}\right)$, whose causality can be arbitrary.
The output equation of state, as before, follows directly form the BG model shown in Fig. 9:

$$
T \dot{S}_{A}=-\left(F_{S}-F_{t}\right) v(t)+T \dot{S}_{Q}
$$

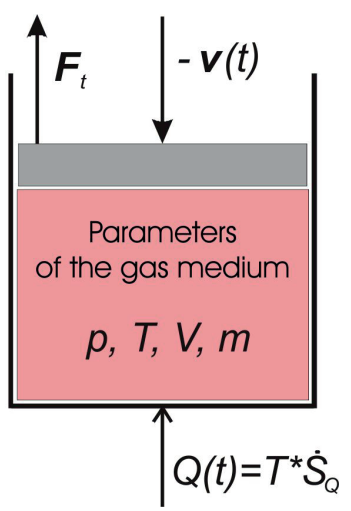

Fig. 8. Diagram of the related systems: mechanical and thermodynamic

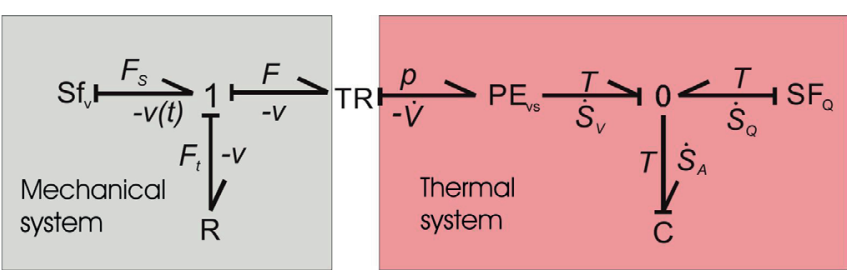

Fig. 9. The BG model of the related systems: mechanical and thermodynamic

Using the basic physical dependence in both of the above examples, the output equations of state (4) and (6) should be presented in the form of (7) [2] enabling simulation research:

$$
\dot{T}=f(T, U)
$$

\section{CONCLUDING REMARKS}

In previous papers $[2,3]$ a new method of modeling thermal processes using the BG method has been presented. It has been shown that the thermal process models can be created without entering new elements into the BG theory such as "Pseudo Bong Graphs" and dual elements of capitalization and distraction. There is also no need to introduce the "gyrator" [1] into the BG models as in the theory of thermodynamics and heat exchange there is no equivalent of this concept, as is the case of mechanical and electrical processes. By creating a BG model covering only thermal processes, there is no possibility of a conflict of causality, mainly due to the existence of only one type of heat energy accumulator. In complex energy systems, the heat processes are associated with the processes of different physical nature, and in this case, the analysis of causality BG model is justified and necessary. 


\section{BIBLIOGRAPHY}

1. Borutzki W.: Bond Graphs a Metodology for Modelling Multidisciplinary Dynamic Systems. Springer, (2010).

2. Cichy M., Kropiwnicki J., Kneba Z.: A Model of Thermal Energy Storage According to the Convention of Bond Graphs (BG) and State Equations (SE). Polish Maritime Research, Vol. 22, nr 4 (88) (2015), pp. 41-47.

3. Cichy M.: Modelowanie systemów energetycznych (Modeling of energetic systems). Wydawnictwo Politechniki Gdańskiej. Gdańsk, (2001), (in Polish).

4. Cieśliński J. T., Mosdorf R.: Gas bubble dynamics experiment and fractal analysis. International Journal of Heat and Mass Transfer Volume 48, Issue 9, (2005), pp. 1808-1818.

5. Creyx M., et al.: Dynamic modelling of the expansion cylinder of an open Joule cycle Ericsson engine: A bond graph approach. Energy 102 (2016), pp. 31-43.

6. Deja M., Siemiątkowski M. S.: Feature-based generation of machining process plans for optimised parts manufacture. Journal of Intelligent Manufacturing (2013), Volume 24, Issue 4, pp. 831-846.

7. Domachowski Z., Dzida M.: Inlet Air Fogging of Marine Gas Turbine in Power Output Loss Compensation. Polish Maritime Research 4 (88) (2015), Vol. 22, pp. 53-58.

8. Hubbard M., Brewer J. W.: Pseudo Bond Graphs of circulating fluids with Application to Solar Heating Design. Journal of the Franklin Institute Vol. 311, No 6, (1981), pp. 339-354.

9. Kaliński K. J., Galewski M. A.: Chatter vibration surveillance by the optimal-linear spindle speed control. Mechanical Systems and Signal Processing Volume 25, Issue 1, (2011), pp. 383-399.

10. Karnopp D. C., Margolis D. L., Rosenberg R. C.: System dynamics: a unified approach. Wiley, New York, (1990).

11. Korczewski Z., Zacharewicz M.: Alternative diagnostic method applied on marine diesel engines having limited monitoring susceptibility. Transactions of the Institute of Measurement and Control. Vol. 34, No. 8 (2012), pp.937-946.

12. Korczewski Z.: Exhaust Gas Temperature Measurements in Diagnostics of Turbocharged Marine Internal Combustion Engines. Part II Dynamic Measurements. Polish Maritime Research 1 (89) (2016) Vol. 23, pp. 68-76.
13. Kortas P., Kropiwnicki J.: Analysis of accumulation possibility of energy dissipated in the braking process of train driven by hybrid locomotive. Combustion Engines, (2015), pp. 631-638.

14. Kowalczyk T., Głuch J., Ziółkowski P.: Analysis of Possible Application of High-Temperature Nuclear Reactors to Contemporary Large-Output Steam Power Plants on Ships. Polish Maritime Research 2 (90) (2016), Vol. 23, pp. 32-41.

15. Kropiwnicki J.: Comparison of energy efficiency of vehicles powered by different fuels. Combustion Engines, nr 3, (2012), pp .34-43.

16. Litwin W., Olszewski A.: Water-Lubricated Sintered Bronze Journal Bearings - Theoretical and Experimental Research. Tribology Transactions. Vol. 57, No. 1 (2014), pp.114-122.

17. M.S. Jha, et al.: Particle filter based hybrid prognostics of proton exchange membrane fuel cell in bond graph framework. Computers and Chemical Engineering 95 (2016), pp. 216-230.

18. Mikielewicz D., Mikielewicz J., Tesmar J.: Improved semiempirical method for determination of heat transfer coefficient in flow boiling in conventional and small diameter tubes. International Journal of Heat and Mass Transfer Volume 50, Issues 19-20, (2007), pp. 3949-3956.

19. Mishra C., Samantaray A.K., Chakraborty G.: Bond graph modeling and experimental verification of a novel scheme for fault diagnosis of rolling element bearings in special operating conditions. Journal of Sound and Vibration 377 (2016), pp. 302-330.

20. Paynter H.M.: Analysis and Design of Engineering Systems. The MIT Press Cambridge, Massachusetts (1961).

21. Sagawa J.K., Nagano M.S., Neto M.S.: A closed-loop model of a multi-station and multi-product manufacturing system using bond graphs and hybrid controllers. European Journal of Operational Research (2016), pp. $1-15$.

22. Shoureshi R., McLaughlin K. M.: Analytical and Experimental Investigation of Flow-Reversibile Heat Exchangers Using Temperature-Entropy Bond Graphs. Journal of Dynamic Systems, Measurement, and Control, Vol. 106 (2), (1984), pp. 170-175.

23. Silva L.I., et al.: Coupling Bond Graph and Energetic Macroscopic Representation for Electric Vehicle Simulation: Mechatronics 24 (2014), pp. 906-913. 
24. Sliwinski P.: The basics of design and experimental tests of the commutation unit of a hydraulic satellite motor. Archives of Civil and Mechanical Engineering, vol. 16, iss. 4 (2016), pp. 634-644.

25. Sosnovsky E., Forget B.: Bond graph representation of nuclear reactor point kinetics and nearly incompressible thermal hydraulics. Annals of Nuclear Energy 68 (2014), pp. 15-29.

26. Thoma J. U., Boumama B. O: Modelling and Simulation in Thermal and Chemical Engineering - a Bond Graph Approach. Springer, (2000).

27. Thoma J. U.: Simulation by Bondgraphs. Springer, Berlin, (1990).

28. Wellstead P.E.: Introduction to System Modeling. Academic Press, London (1979).

\section{CONTACT WITH THE AUTHOR}

\section{Jacek Kropiwnicki}

Gdańsk University of Technology Faculty of Mechanical Engineering

11/12 Narutowicza St. 80 - 233 Gdańsk

Poland 